\title{
Social determinants of awareness and behavior regarding STDs and HIV/AIDS among ever married women in Bangladesh
}

JUWEL RANA A-F

Department of Sociology, Hajee Mohammad Danesh Science \& Technology University and South Asian Youth Research Institute for Development (SAYRID), Bangladesh

A - Study Design, B - Data Collection, C - Statistical Analysis, D - Data Interpretation, E - Manuscript Preparation, $\mathbf{F}$ - Literature Search, $\mathbf{G}$ - Funds Collection

Summary Background. About 8,900 people are living with HIV/AIDS, and 1,000 AIDS-related deaths had been reported in Bangladesh by the end of 2014 .

Objectives. The study investigates the social determinants of awareness and behavior regarding STDs and HIV/AIDS among ever married women in Bangladesh.

Material and methods. This cross-sectional research extracted data concerning 17,828 ever married women from the Bangladesh Demographic and Health Survey (BDHS) in 2014. The chi-square $\left(\chi^{2}\right)$ and multinomial logistic regression model were used to identify the factors associated with knowledge, awareness and behavior concerning STDs and HIV/AIDS.

Results. Overall, $28.6 \%$ of examined ever married women have never heard of STDs or HIV/AIDS nor any of their prevention methods. Also, only $15.6 \%$ of reported women were the decision makers regarding the use of contraception during sexual intercourse, and $91.3 \%$ of women had the capacity to refuse sexual contact with their STD-infected husband/partner. Women who belong to households classified as lower class $(\mathrm{OR}=0.525,95 \% \mathrm{Cl}=0.461-0.598)$ or middle class $(\mathrm{OR}=0.643$, $95 \% \mathrm{Cl}=0.564-0.733)$ had less comprehensive knowledge and awareness of STDs and HIV/AIDS than those categorized as upper class. Women at a level of education below secondary $(\mathrm{OR}=0.200,95 \% \mathrm{Cl}=0.179-0.223)$ also had less comprehensive knowledge and awareness than highly educated women. Moreover, women living in an urban residence $(\mathrm{OR}=1.141$, $95 \% \mathrm{Cl}=1.003-1.297)$ were more likely to make the decision of using contraception and $(\mathrm{OR}=1.546,95 \% \mathrm{Cl}=1.351-1.770)$ more likely to refuse sexual contact with an STD-infected husband/partner than their rural counterparts. Formally unemployed women $(\mathrm{OR}=0.894,95 \% \mathrm{Cl}=0.793-1.010)$ were less likely to refuse sexual intercourse with an STD-infected husband than employed women.

Conclusions. Social determinants such as education, wealth and media exposure determine the level of knowledge and awareness and affect the behavior of women concerning STDs and HIV/AIDS.

Key words: STDs, HIV/AIDS, knowledge and awareness, behavior, social determinants.

Rana J. Social determinants of awareness and behavior regarding STDs and HIV/AIDS among ever married women in Bangladesh. Fam Med Prim Care Rev 2016; 18(4): 460-461, doi: 10.5114/fmpcr.2016.63702.

\section{Background}

Every day about one million patients with curable sexually transmitted diseases (STDs) infected globally, of which $50 \%$ affect people under 25 years of age [1]. According to the Joint United Nations Programme on HIV/AIDS (UNAIDS), about 8,900 people are living with HIV/AIDS, and about 1,000 AIDS-related deaths had been reported in Bangladesh by the end of 2014. Moreover, 1,000 new HIV infections were noted in 2014 [2]. The data collected between 1990 and 2014 showed that of 88.799 million Bangladeshi residents aged 15-49 years, 8,600 adults (15+) were living with HIV/AIDS, including 2,900 adult females (15+). The prevalence rate of HIV infection among adults aged $15-49$ years is around $0.01 \%[2,3]$, seven in ten ever married women aged 15-49 have heard about STDs and HIV/ /AIDS. The Bangladesh Demographic and Health Survey 2014 (BDHS) [4] showed that the knowledge and awareness of STDs and HIV/AIDS remained unchanged between 2011 and 2014. Thus, it is imperative to pay attention to social factors, as they determine the level of knowledge and awareness of STDs and HIV/AIDS among ever married women aged 15-49 years in Bangladesh, as well as influence their sexual behavior.

In patients infected with STDs, the risk of developing other sexually transmitted diseases, such as chlamydiasis, gonorrhea, genital herpes, human papillomavirus (HPV), AIDS and syphilis, as well as other reproductive tract infections, is higher $[5,6]$. The World Health Organization (WHO) recognized AIDS as the second leading cause of death among adolescents and pointed to the rising number of AIDS-related mortality [7]. Global AIDS deaths are projected to rise to 6.5 million per year in 2030, making it the number one cause of death or disability-adjusted life years (DALYs) [8]. HIV/AIDS is particularly prevalent in low and middle income countries; UNAIDS estimated an $11 \%$ increase in AIDS-related deaths in Asia and the Pacific between 2000 and 2014 [2]. Women and adolescents are the most vulnerable to STDs and HIV/AIDS [9-12].

The overall prevalence of HIV/AIDS in Bangladesh is lower than in any other Asian country, yet Bangladeshi women and adolescents are still in high-risk groups for STDs and HIV/AIDS. The risk factors include geographical location, high incidence of sexually transmitted infections among 
vulnerable groups, women marrying migrant men, lack of proper knowledge and awareness about STDs and HIV/AIDS [9-14]. The study found that the knowledge and awareness of HIV/AIDS prevention among the general population is unsatisfactory. As many as $40 \%$ of ever married women have never heard of HIV/AIDS, and only 19\% have heard about it, but know nothing about any HIV prevention methods. Only $41 \%$ of women knew about one or more correct ways to prevent STDs and HIV/AIDS [15]. According to another study, $29.4 \%$ of ever married women did not have any knowledge and awareness about STDs. Among all the respondents, $38.2 \%$ of women living in urban settings and $32.2 \%$ of rural women showed some knowledge and awareness about STDs. The study also demonstrated that a higher level of education increased the knowledge of STDs, and older women are generally more aware of STDs than younger ones [16].

The study showed that garment workers $(76.9 \%)$ had poor knowledge of STDs and HIV/AIDS, yet their knowledge tended to increase significantly with age. The difference in knowledge and awareness between male and female garment workers was insignificant. Female garment workers with secondary education were more knowledgeable about STDs and HIV/AIDS than their less educated counterparts [17]. The outdated social system and health care service are the leading causes of poor knowledge and awareness about STDs and HIV/AIDS, particularly in the younger age group ( $<29$ years), because they are perceived neither as children nor as adults [16]. A study carried out in a Turkish Cypriot (TC) community revealed that $42.32 \%$ of respondents described school as the main source of awareness about STDs, and only $7.57 \%$ of the group cited health care professionals as the source of information [18]. Another study that was conducted in Bangladesh asserted that media exposure along with women's education, age, employment and urban residence are the significant predictors of their knowledge and awareness of STDs and HIV/AIDS [19]. The results derived from the studies bring into question of which social factors affect the level of knowledge and awareness regarding STDs and HIV/AIDS and their sexual behavior among ever married women.

Commercial sex workers with their clients, truck drivers and drug users are high risk groups due to exhibiting STDs or HIV/AIDS-related risk behaviors such as unsafe sexual intercourse and sharing syringes for drug intake [13, 20, 21]. Despite recent advances in biomedical sciences and the development of cures and treatments for infectious diseases, human pathogens continue to emerge or re-emerge today, especially those affecting socially and economically underprivileged groups. In this regard, social epidemiology, which emerged from the proponents of social medicine, argues for greater consideration of social and ecological factors in diagnosis of the disease [22-29]. Farmer claimed that social inequalities are responsible for the recent emergence of infections such as Ebola, AIDS and tuberculosis [30]. Sir Michael Marmot, chair of the WHO Commission on Social Determinants of Health, has proposed a framework for considering the causation of diseases through a hierarchy of social causes based on ten solid facts, which include the social gradient, stress, early life, social exclusion, work, unemployment, social support, addiction, food and transport $[31,32]$. He emphasized that social determinants must focus on material conditions and control of infectious diseases as well [31]. Social epidemiologists argue that whether or not we suffer from and survive an infectious or chronic disease is heavily dependent on our place in society [33]. Therefore, Farmer and other social epidemiologists have strongly encouraged the social determinants approach to be used in order to study and prevent STDs and HIV/AIDS [30, 34, 35].

Most of the STD and HIV/AIDS-related studies in Bangladesh are typically concentrated on biomedical and be- havioral risk factors among commercial sex workers and their clients, truck drivers and drug users and focus only on their knowledge of HIV/AIDS. Social determinants of their level of knowledge, awareness and behavior, especially among women, have been largely ignored in STD and HIV/ /AIDS-related studies [36]. Currently, the world is embarking on a fast track approach to halt and reverse the spread of STDs and the AIDS epidemic by 2030 as part of Sustainable Development Goals (SDGs) [37]. Bangladesh has done a tremendous job attaining the Millennium Development Goals (MDGs), MDG 6: combat HIV/AIDS still remains an unfinished agenda.

\section{Objectives}

The study investigates the social determinants of awareness and behavior regarding STDs and HIV/AIDS among ever married women in Bangladesh.

\section{Material and methods}

The study conducted, extracted data of 17,828 ever married women from the nationally representative cross-sectional Bangladesh Demographic and Health Survey (BDHS) 2014. The selection of sociodemographic variables associated with awareness of STDs and HIV/AIDS and sexual behavior was guided by previous studies and the researcher's knowledge in this field [10-36]. Originally, coded variables were used in the study, and some variables were attained as a combination of three or more variables and regrouping the levels of specific variables.

The dependent variable, the level of knowledge and awareness of STDs and HIV/AIDS, was created with several combinations. The value 'none' refers to: never heard of STDs and HIV/AIDS and do not know any of their prevention methods, while 'poor' refers to: at least heard about STDs and HIV/AIDS and know some of their prevention methods. The value 'moderate' means: heard of both STDs and HIV/AIDS and know some of their prevention methods, and 'comprehensive' means: heard of both STDs and HIV/ /AIDS and know both of the prevention methods. Use of contraceptives and refusing sex with an STD-infected husband were also outcome variables of women's behavior regarding STD and HIV/AIDS. To delineate the STD and HIV/AIDS-preventive behavior of women, 'other' and 'don't know' answers were excluded from questions on the decision maker for using contraception and right to refuse sex with an STD-infected husband, respectively.

In this study, social determinants include age, marital status, division, place of residence, education of women and their husband/partner, exposure to mass media, religion, use of contraception, working status, economic status and their belonging to income generating organization (IGO). Education of women and their partner/husband were categorized as follows: below secondary (No Education and Primary) and secondary+ (Secondary and Higher). The variable 'exposure to mass media' was generated by taking into account various combinations of the frequency of reading newspapers and magazines, listening to the radio and watching television. Women were characterized as those who have accessed or who have not accessed any form of mass media (newspaper or magazine, radio and television). Economic status was categorized as lower class (the poorest and the poor), middle class (middle income) and upper class (the rich and the richest). Grameen Bank, Association for Social Advancement (ASA), PROSHIKA, Bangladesh Rural Advancement Committee (BRAC) and Bangladesh Rural Development Board (BRDB) were considered as IGOs, and women were categorized as those affiliated or those non-affiliated with any of these agencies (Figure 1). 


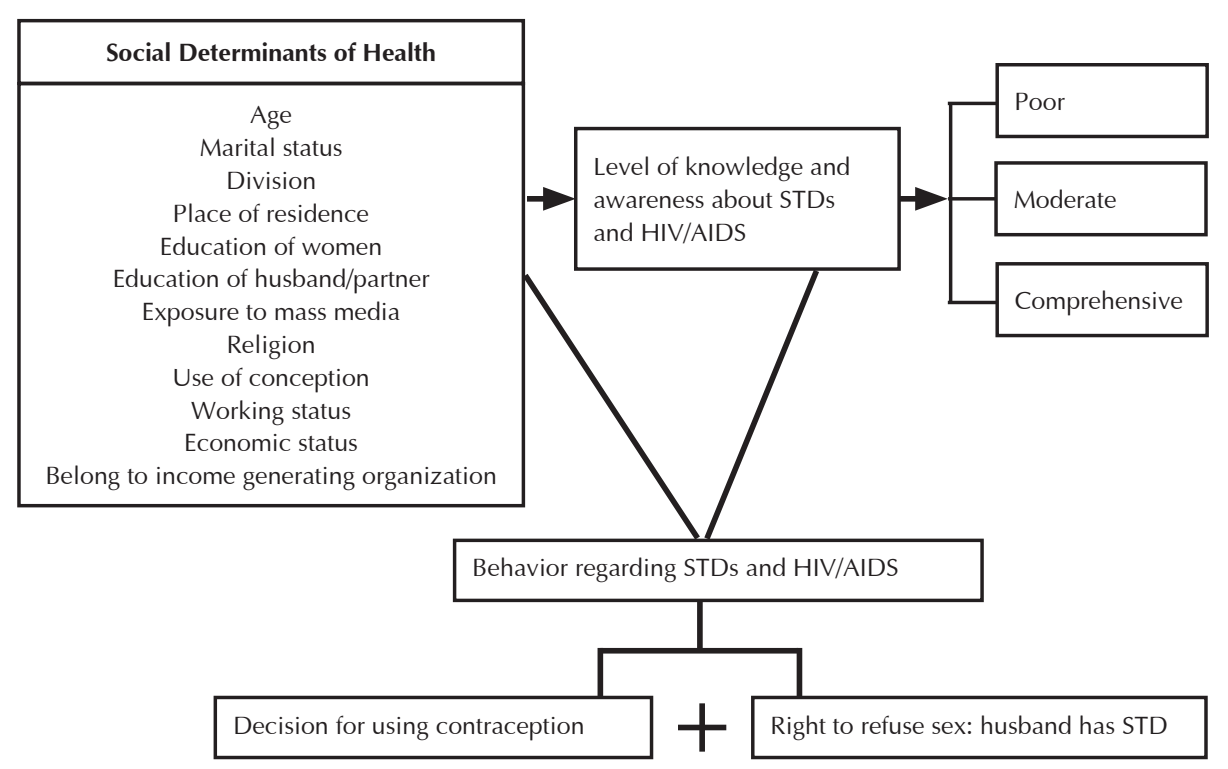

Figure 1. Conceptual framework

A total of 15 explanatory variables were used as categorical in the present investigation. In exploring the association between independent variables and the level of knowledge and awareness of STDs and HIV/AIDS, as well as women's STD and HIV/AIDS-related behavior, both bivariate and multivariate analyses were performed (presented in Tables 1-4). The multivariate analysis was conducted using multinomial logistic regression, a model which is most appropriate for a polytomous dependent variable [38]. The Statistical Package for the Social Sciences (SPSS-20) for Windows version 10 was employed to execute a statistical analysis of the data.

\section{Results}

\section{Knowledge and awareness}

The results of the study, shown in Table 1, indicate that $28.6 \%$ of ever married women in Bangladesh have never heard of STDs and HIV/AIDS or any of their prevention methods. The findings suggest that there was no improvement in the level of knowledge and awareness of STDs and HIV/AIDS among women during the period 2007-2011, when a similar study was conducted [4]. $12.7 \%, 24.3 \%$, and
$34.3 \%$ women had poor, moderate and higher knowledge and awareness about STDs and HIV/AIDS, respectively. The findings and the associated chi-square $\left(\chi^{2}\right)$ test presented in Table 1 reveal that the level of knowledge and awareness about STDs and HIV/AIDS in Bangladesh was significantly associated with all social determinants. All the social determinants were also included in the multivariate setting.

The results presented in Table 2 show Odds Ratios (ORs) and their associated confidence interval $(95 \% \mathrm{Cl})$ for each categorical variable included in the multinomial logistic regression model. According to the fitted model, a respondent's division, place of residence, education of women and their partner/husband, exposure to mass media, religious affiliation, use of contraception, working and economic status had a statistically significant effect on poor knowledge and awareness of STDs and HIV/AIDS among women in Bangladesh. Their age, marital status and affiliation with IGOs lose their statistical significance once the effects of other variables were controlled. When other variables were controlled for, all of the selected variables appeared as significant predictors of the moderate knowledge and awareness of STDs and HIV/AIDS among the women in Bangladesh, except affiliation with IGOs (Table 2).

\begin{tabular}{|c|c|c|c|c|c|c|}
\hline \multirow[t]{2}{*}{ Social determinants } & \multirow[t]{2}{*}{ Levels } & \multicolumn{5}{|c|}{ (\%) Level of knowledge and awareness about STDs \& HIV } \\
\hline & & None $^{\mathrm{a}}$ & Poor ${ }^{b}$ & Moderate $^{c}$ & Comprehensive ${ }^{\mathrm{d}}$ & Total \\
\hline \multirow[t]{3}{*}{$\mathrm{Age}^{* * *}$} & $15-29$ years & 23.8 & 13.4 & 26.3 & 36.6 & $5,172(29.0)$ \\
\hline & 30-39 years & 31.2 & 12.3 & 23.3 & 33.2 & $7,311(41.0)$ \\
\hline & $40-49$ years & 29.9 & 12.7 & 23.9 & 33.5 & $5,345(30.0)$ \\
\hline \multirow[t]{2}{*}{ Marital status $^{* * *}$} & married & 27.9 & 12.7 & 24.7 & 34.8 & $16,797(94.2)$ \\
\hline & separated/divorced & 41.4 & 14.2 & 19.2 & 25.2 & $1,031(5.8)$ \\
\hline \multirow[t]{7}{*}{ Division $^{* * *}$} & Barisal & 25.5 & 13.0 & 27.8 & 33.7 & $2,140(12.0)$ \\
\hline & Chittagong & 28.2 & 15.9 & 27.2 & 28.6 & $2,857(16.0)$ \\
\hline & Dhaka & 21.4 & 11.6 & 24.2 & 42.9 & $3,089(17.3)$ \\
\hline & Khulna & 20.3 & 13.2 & 28.4 & 38.1 & $2,575(14.4)$ \\
\hline & Rajshahi & 32.6 & 10.3 & 21.8 & 35.3 & $2,511(14.1)$ \\
\hline & Rangpur & 37.3 & 10.3 & 18.2 & 34.2 & $2523(14.2)$ \\
\hline & Sylhet & 38.0 & 15.1 & 22.6 & 24.3 & $2,133(12.0)$ \\
\hline
\end{tabular}




\begin{tabular}{|c|c|c|c|c|c|c|}
\hline \multirow[t]{2}{*}{ Social determinants } & \multirow[t]{2}{*}{ Levels } & \multicolumn{5}{|c|}{ (\%) Level of knowledge and awareness about STDs \& HIV } \\
\hline & & None $^{\mathrm{a}}$ & Poor $^{b}$ & Moderate $^{\mathrm{c}}$ & Comprehensive $^{d}$ & Total \\
\hline \multirow[t]{2}{*}{ Place of residence ${ }^{* * *}$} & urban & 16.5 & 12.3 & 27.1 & 44.1 & $6,158(34.5)$ \\
\hline & rural & 35.1 & 13.0 & 22.9 & 29.1 & $11,670(65.5)$ \\
\hline \multirow[t]{2}{*}{ Education of women ${ }^{* * *}$} & below secondary & 45.3 & 13.0 & 19.5 & 22.2 & 9,409 (52.8) \\
\hline & secondary+ & 10.0 & 12.5 & 29.7 & 47.8 & $8,419(47.2)$ \\
\hline \multirow{2}{*}{$\begin{array}{l}\text { Education of husband/ } \\
\text { /partner }^{* * *}\end{array}$} & below secondary & 41.9 & 12.8 & 21.0 & 24.3 & 9,901 (55.5) \\
\hline & secondary+ & 12.1 & 12.7 & 28.5 & 46.7 & $7,924(44.5)$ \\
\hline \multirow[t]{2}{*}{ Exposure to mass media ${ }^{* * *}$} & no access & 51.7 & 11.8 & 16.8 & 19.8 & $6,580(36.9)$ \\
\hline & access & 15.2 & 13.3 & 28.8 & 42.8 & $11,248(63.1)$ \\
\hline \multirow[t]{2}{*}{ Religion $^{* * *}$} & muslim & 28.1 & 12.9 & 24.6 & 34.4 & $16,107(90.3)$ \\
\hline & other & 33.6 & 11.7 & 21.7 & 32.9 & $1,721(9.7)$ \\
\hline \multirow[t]{2}{*}{ Use of contraceptive ${ }^{* * *}$} & not using & 32.1 & 13.0 & 23.7 & 31.1 & $7,378(41.4)$ \\
\hline & using any method & 26.2 & 12.5 & 24.8 & 36.5 & $10,450(58.6)$ \\
\hline \multirow[t]{2}{*}{ Working status $^{* * *}$} & not working & 27.4 & 13.7 & 24.8 & 34.1 & $12,211(68.5)$ \\
\hline & working & 31.3 & 10.7 & 23.4 & 34.6 & $5,617(31.5)$ \\
\hline \multirow[t]{3}{*}{ Economic status $^{* * *}$} & lower class & 48.6 & 12.1 & 17.2 & 22.1 & $6,599(37.0)$ \\
\hline & middle class & 27.2 & 13.6 & 27.2 & 32.0 & $3,611(20.3)$ \\
\hline & upper class & 12.0 & 12.9 & 29.1 & 45.9 & $7,618(42.7)$ \\
\hline \multirow[t]{2}{*}{ Belong to IGO ${ }^{\mathrm{e}^{* *}}$} & not affiliated & 28.2 & 12.6 & 24.3 & 34.9 & $13,480(75.5)$ \\
\hline & affiliated & 29.9 & 13.2 & 24.4 & 32.4 & $4,383(24.5)$ \\
\hline \multicolumn{2}{|l|}{ Overall } & 28.6 & 12.7 & 24.3 & 34.3 & $17,828(100.0)$ \\
\hline
\end{tabular}

\begin{tabular}{|c|c|c|c|c|}
\hline \multirow[t]{2}{*}{$\begin{array}{l}\text { Social determi- } \\
\text { nants }\end{array}$} & \multirow[t]{2}{*}{ Levels } & $\begin{array}{l}\text { Poor knowledge and } \\
\text { awareness }\end{array}$ & $\begin{array}{l}\text { Moderate knowledge and } \\
\text { awareness }\end{array}$ & $\begin{array}{l}\text { Comprehensive knowl- } \\
\text { edge and awareness }\end{array}$ \\
\hline & & $\begin{array}{l}\text { Odds Ratio (95\% } \\
\text { Confidence Interval) }\end{array}$ & $\begin{array}{l}\text { Odds Ratio ( } 95 \% \\
\text { Confidence Interval) }\end{array}$ & $\begin{array}{l}\text { Odds Ratio (95\% } \\
\text { Confidence Interval) }\end{array}$ \\
\hline \multirow[t]{3}{*}{ Age } & 15-29 years & $0.906(0.785-1.046)$ & $0.882(0.778-1.000)^{* *}$ & $0.842(0.745-0.951)^{* *}$ \\
\hline & 30-39 years & $0.919(0.810-1.042)$ & $0.927(0.831-1.034)$ & $0.956(0.860-1.062)$ \\
\hline & 40-49 years $(\mathrm{RC})$ & 1.000 & 1.000 & 1.000 \\
\hline \multirow[t]{2}{*}{ Marital status } & married & $1.005(0.807-1.251)$ & $1.404(1.147-1.717)^{* * *}$ & $1.376(1.134-1.670)^{* * *}$ \\
\hline & $\begin{array}{l}\text { separated/divorced } \\
\text { (RC) }\end{array}$ & 1.000 & 1.000 & 1.000 \\
\hline \multirow[t]{7}{*}{ Division } & Barisal & $1.264(1.030-1.552)^{* *}$ & $1.786(1.490-2.141)^{* * *}$ & $1.892(1.577-2.270)^{* * *}$ \\
\hline & Chittagong & $0.900(0.749-1.082)$ & $0.889(0.751-1.053)$ & $0.800(0.674-0.949)^{* *}$ \\
\hline & Dhaka & $0.982(0.809-1.192)$ & $1.198(1.008-1.424)^{* *}$ & $1.819(1.536-2.153)^{* * *}$ \\
\hline & Khulna & $1.363(1.118-1.662)^{* *}$ & $1.806(1.513-2.157)^{* * *}$ & $2.107(1.766-2.514)^{* * *}$ \\
\hline & Rajshahi & $0.599(0.489-0.734)^{* * *}$ & $0.747(0.626-0.892)^{* * *}$ & $1.042(0.877-1.238)$ \\
\hline & Rangpur & $0.629(0.515-0.769)^{* * *}$ & $0.704(0.589-0.842)^{* * *}$ & $1.144(0.964-1.357)$ \\
\hline & Sylhet (RC) & 1.000 & 1.000 & 1.000 \\
\hline \multirow[t]{2}{*}{ Place of residence } & urban & $1.205(1.061-1.368)^{* *}$ & $1.318(1.181-1.472)^{* * *}$ & $1.556(1.400-1.729)^{* * *}$ \\
\hline & rural (RC) & 1.000 & 1.000 & 1.000 \\
\hline \multirow{2}{*}{$\begin{array}{l}\text { Education of } \\
\text { women }\end{array}$} & below secondary & $0.375(0.329-0.427)^{* * *}$ & $0.260(0.232-0.292)^{* * *}$ & $0.200(0.179-0.223)^{* * *}$ \\
\hline & secondary+ (RC) & 1.000 & 1.000 & 1.000 \\
\hline \multirow{2}{*}{$\begin{array}{l}\text { Education of hus- } \\
\text { band/partner }\end{array}$} & below secondary & $0.611(0.538-0.695)^{* * *}$ & $0.577(0.516-0.645)^{* * *}$ & $0.466(0.419-0.518)^{* * *}$ \\
\hline & secondary+ (RC) & 1.000 & 1.000 & 1.000 \\
\hline \multirow{2}{*}{$\begin{array}{l}\text { Exposure to mass } \\
\text { media }\end{array}$} & no access & $0.413(0.365-0.469)^{* * *}$ & $0.338(0.303-0.377)^{* * *}$ & $0.318(0.286-0.353)^{* * *}$ \\
\hline & access (RC) & 1.000 & 1.000 & 1.000 \\
\hline
\end{tabular}


Table 2. Multinomial logistic regression model predictions of Odds Ratios (ORs) and Confidence Intervals (CI) for association between social determinants and level of knowledge and awareness about STDs \& HIV

\begin{tabular}{|c|c|c|c|c|}
\hline \multirow[t]{2}{*}{$\begin{array}{l}\text { Social determi- } \\
\text { nants }\end{array}$} & \multirow[t]{2}{*}{ Levels } & $\begin{array}{l}\text { Poor knowledge and } \\
\text { awareness }\end{array}$ & $\begin{array}{l}\text { Moderate knowledge and } \\
\text { awareness }\end{array}$ & $\begin{array}{l}\text { Comprehensive knowl- } \\
\text { edge and awareness }\end{array}$ \\
\hline & & $\begin{array}{l}\text { Odds Ratio ( } 95 \% \\
\text { Confidence Interval) }\end{array}$ & $\begin{array}{l}\text { Odds Ratio ( } 95 \% \\
\text { Confidence Interval) }\end{array}$ & $\begin{array}{l}\text { Odds Ratio ( } 95 \% \\
\text { Confidence Interval) }\end{array}$ \\
\hline \multirow[t]{2}{*}{ Religion } & muslim & $1.360(1.136-1.628)^{* * *}$ & $1.471(1.257-1.721)^{* * *}$ & $1.421(1.224-1.649)^{* * *}$ \\
\hline & other (RC) & 1.000 & 1.000 & 1.000 \\
\hline \multirow{2}{*}{$\begin{array}{l}\text { Use of contracep- } \\
\text { tive }\end{array}$} & not using & $0.803(0.717-0.900)^{* * *}$ & $0.801(0.725-0.884)^{* * *}$ & $0.747(0.679-0.822)^{* * *}$ \\
\hline & $\begin{array}{l}\text { using any method } \\
\text { (RC) }\end{array}$ & 1.000 & 1.000 & 1.000 \\
\hline \multirow[t]{2}{*}{ Working status } & not working & $1.177(1.046-1.326)^{* *}$ & $0.914(0.826-1.010)^{*}$ & $0.842(0.765-0.927)^{* * *}$ \\
\hline & working (RC) & 1.000 & 1.000 & 1.000 \\
\hline \multirow{3}{*}{ Economic status } & lower class & $0.641(0.548-0.749)^{* * *}$ & $0.506(0.442-0.580)^{* * *}$ & $0.525(0.461-0.598)^{* * *}$ \\
\hline & middle class & $0.762(0.652-0.890)^{* * *}$ & $0.741(0.648-0.847)^{* * *}$ & $0.643(0.564-0.733)^{* * *}$ \\
\hline & upper class (RC) & 1.000 & 1.000 & 1.000 \\
\hline \multirow[t]{2}{*}{ Belong to IGO } & not affiliated & $1.010(0.902-1.131)$ & $1.057(0.963-1.160)$ & $1.139(1.045-1.242)^{* *}$ \\
\hline & affiliated (RC) & 1.000 & 1.000 & 1.000 \\
\hline
\end{tabular}

Based on this analysis, age is the most significant factor in determining comprehensive knowledge and awareness about STDs and AIDS among women in Bangladesh. The results suggest that women aged 15-29 years had 0.842 times less comprehensive knowledge and awareness about STDs and AIDS than women aged 40-49 years. Women who had a husband/partner were 1.376 times more likely to have comprehensive knowledge and awareness about STDs and HIV/AIDS than women who did not have a husband/ /partner. Women living in the Barisal, Dhaka and Khulna divisions had 1.892, 1.819 and 2.107 times more comprehensive knowledge and awareness, respectively, than females from the Sylhet division.

Women residing in Chittagong had less comprehensive knowledge and awareness than women from the Sylhet division. Women living in urban areas had $56 \%(\mathrm{OR}=1.556$, $95 \% \mathrm{Cl}=1.400-1.729)$ more comprehensive understanding of STDs and HIV/AIDS than women living in the countryside. In other words, age and geographical location were proven to be important determinants of comprehensive knowledge and awareness of STDs and HIV/AIDS among women.

Women whose education level was below secondary $(\mathrm{OR}=0.200,95 \% \mathrm{Cl}=0.179-0.223)$ had less comprehensive knowledge and awareness of STDs and HIV/AIDS than women who had secondary+ education. Correspondingly, women whose partner's/husband's education level was below secondary also exhibited less comprehensive knowledge and awareness of STDs and HIV/AIDS than women whose partner/husband had secondary+ education. The results showed that women's knowledge and awareness increases with the level of education for both women and their partner/husband.

Lack of access to mass media was an important indicator of the level of knowledge and awareness about STDs and HIV/AIDS among women. Women who did not have exposure to mass media had 0.318 times less comprehensive knowledge and awareness about STDs and HIV/AIDS than the women who had exposure to mass media. Also, women who were not using contraception $(\mathrm{OR}=0.747,95 \% \mathrm{Cl}=$ 0.679-0.822) were more likely to have less comprehensive knowledge and awareness about STDs and HIV/AIDS than women who used some contraceptive method. Moreover, Muslim women had 1.421 times more holistic knowledge and awareness about STDs and HIV/AIDS than women who were practicing other religions.

Work and economic status of women are important factors affecting their knowledge and awareness about STDs and HIV/AIDS. Unemployed women $(\mathrm{OR}=0.842,95 \% \mathrm{Cl}$ $=0.765-0.927)$ tended to have less comprehensive knowledge and awareness than women who were employed. Also, women from lower- $(\mathrm{OR}=0.525,95 \% \mathrm{Cl}=0.461-0.598)$ and middle-class $(\mathrm{OR}=0.643,95 \% \mathrm{Cl}=0.564-0.733$ ) households were more likely to have less comprehensive knowledge and awareness than women from upper-class families. Women who were not affiliated with IGOs had $13.9 \%(\mathrm{OR}=1.139,95 \% \mathrm{Cl}=1.045-1.242)$ more comprehensive knowledge and awareness than women who were affiliated.

\section{Behavior regarding STDs and HIV/AIDS}

The results shown in Table 3 suggest that only $15.6 \%$ of women were decision makers regarding the use of contraception during sexual intercourse. $91.3 \%$ of women had the capacity to refuse sex with their STD-infected husband/partner, which indicates the risk-free sexual behavior of women. The results and associated chi-square $\left(\chi^{2}\right)$ test disclosed that the decision pertaining using contraception is strongly related to age, division, education of women and their partner/husband, exposure to mass media, working status, economic situation and their knowledge and awareness about STDs and HIV/AIDS. On the contrary, the marital status of women, place of residence, religion, use of contraception and their association with IGOs appeared to have no impact on the decision regarding contraception use.

Table 3 also shows that division, place of residence, education of women and their husband/partner, exposure to mass media, use of contraception, working status, economic status and association with IGOs were significant predictors of a woman s right to refuse sex with an STD-infected husband. Moreover, women's knowledge and awareness of STDs and HIV/AIDS are closely connected to their right to refuse sex with an STD-infected husband. On the other hand, women's age, marital status and their religion seemed to have no impact on their right to refuse sex with an STD-infected husband. Hence, all these social determinants were also considered in a multivariate setting. 


\begin{tabular}{|c|c|c|c|c|c|c|c|c|}
\hline \multirow[t]{2}{*}{$\begin{array}{l}\text { Social determi- } \\
\text { nants }\end{array}$} & \multirow[t]{2}{*}{ Levels } & \multicolumn{4}{|c|}{ (\%) Decision maker for using contraception ${ }^{\mathrm{a}}$} & \multicolumn{3}{|c|}{$\begin{array}{l}\text { (\%) Right to refuse sex: } \\
\text { husband has STD }\end{array}$} \\
\hline & & $\begin{array}{l}\text { Respon- } \\
\text { dent }\end{array}$ & $\begin{array}{l}\text { Husband } \\
\text { /Partner }\end{array}$ & $\begin{array}{l}\text { Joint } \\
\text { decision }\end{array}$ & Total & No & Yes & Total \\
\hline \multirow[t]{3}{*}{$\operatorname{Age}^{\mathrm{a}^{* * *}}$} & $15-29$ years & 12.1 & 10.3 & 77.5 & $2,841(27.3)$ & 8.8 & 91.2 & $5,126(29.0)$ \\
\hline & 30-39 years & 16.4 & 8.9 & 74.7 & 3,839 (36.9) & 8.9 & 91.1 & $7,239(41.0)$ \\
\hline & $40-49$ years & 17.4 & 8.5 & 74.1 & $3,732(35.8)$ & 8.5 & 91.5 & $5,297(30.0)$ \\
\hline \multirow[t]{2}{*}{ Marital status } & married & 15.6 & 9.2 & 75.2 & $10,412(100)$ & 8.7 & 91.3 & $16,658(94.3)$ \\
\hline & $\begin{array}{l}\text { separated } \\
\text { /divorced }\end{array}$ & - & - & - & - & 9.0 & 91.0 & $1,004(5.7)$ \\
\hline \multirow[t]{7}{*}{ Division $^{\mathrm{ab}^{* * *}}$} & Barisal & 20.0 & 11.6 & 68.4 & $1,254(12.0)$ & 10.9 & 89.1 & $2,124(12.0)$ \\
\hline & Chittagong & 11.8 & 8.7 & 72.8 & $1,451(13.9)$ & 8.5 & 91.5 & $2,811(15.9)$ \\
\hline & Dhaka & 13.7 & 9.1 & 77.2 & $1,824(17.5)$ & 6.8 & 93.2 & 3,045 (17.2) \\
\hline & Khulna & 18.4 & 8.7 & 72.8 & $1,614(15.5)$ & 6.4 & 93.6 & $2,568(14.5)$ \\
\hline & Rajshahi & 14.3 & 7.5 & 78.2 & 1,657 (15.9) & 4.0 & 96.0 & $2,494(14.1)$ \\
\hline & Rangpur & 16.1 & 7.6 & 76.4 & $1,661(16.0)$ & 13.2 & 86.8 & $2,508(14.2)$ \\
\hline & Sylhet & 15.5 & 14.0 & 70.5 & $951(9.1)$ & 12.8 & 87.2 & $2,112(12.0)$ \\
\hline \multirow{2}{*}{$\begin{array}{l}\text { Place of resi- } \\
\text { dence } \text { b }^{b^{* * *}}\end{array}$} & urban & 14.9 & 9.1 & 76.0 & 3,739 (35.9) & 5.9 & 94.1 & $6,117(34.6)$ \\
\hline & rural & 16.0 & 9.2 & 74.8 & $6,673(64.1)$ & 10.3 & 89.7 & $11,545(65.4)$ \\
\hline \multirow{2}{*}{$\begin{array}{l}\text { Education of } \\
\text { women }^{\text {ab }}\end{array}$} & below secondary & 19.4 & 9.4 & 71.2 & $5,341(51.3)$ & 9.7 & 90.3 & $9,281(52.5)$ \\
\hline & secondary+ & 11.6 & 8.9 & 79.5 & $5,071(48.7)$ & 7.6 & 92.4 & $8,381(47.5)$ \\
\hline \multirow{2}{*}{$\begin{array}{l}\text { Education of } \\
\text { husband/ } \\
/ \text { partner }^{\text {ab*** }}\end{array}$} & below secondary & 18.9 & 9.2 & 72.0 & $5,799(55.7)$ & 9.4 & 90.6 & $9,774(55.3)$ \\
\hline & secondary+ & 11.5 & 9.2 & 79.4 & $4,613(44.3)$ & 7.9 & 92.1 & $7,885(44.7)$ \\
\hline \multirow{2}{*}{$\begin{array}{l}\text { Exposure to mass } \\
\text { media }^{\mathrm{ab}^{* * *}}\end{array}$} & no access & 18.1 & 10.8 & 71.0 & $3,679(35.3)$ & 11.4 & 88.6 & $6,475(36.7)$ \\
\hline & access & 14.2 & 8.2 & 77.6 & $6,733(64.7)$ & 7.2 & 92.8 & $11,187(63.3)$ \\
\hline \multirow[t]{2}{*}{ Religion } & muslim & 15.6 & 9.3 & 75.1 & 9,311 (89.4) & 8.7 & 91.3 & $15,956(90.3)$ \\
\hline & others & 15.2 & 8.4 & 76.5 & $1,101(10.6)$ & 9.5 & 90.5 & $1,706(9.7)$ \\
\hline \multirow{2}{*}{$\begin{array}{l}\text { Use of contra- } \\
\text { ceptive }^{b^{* * *}}\end{array}$} & not using & 15.6 & 9.2 & 75.2 & $10,412(100.0)$ & 9.6 & 90.4 & $7,278(41.2)$ \\
\hline & $\begin{array}{l}\text { using any } \\
\text { method }\end{array}$ & - & - & - & - & 8.2 & 91.8 & $10,384(58.8)$ \\
\hline \multirow{2}{*}{$\begin{array}{l}\text { Working } \\
\text { status }^{\mathrm{a}^{* * *} \mathrm{~b}^{* *}}\end{array}$} & not working & 14.7 & 10.2 & 75.1 & $6,926(66.5)$ & 9.1 & 90.9 & $12,087(68.4)$ \\
\hline & working & 17.3 & 7.1 & 75.6 & $3,486(33.5)$ & 7.9 & 92.1 & $5,575(31.6)$ \\
\hline \multirow{3}{*}{$\begin{array}{l}\text { Economic } \\
\text { status }^{\mathrm{ab}^{* * *}}\end{array}$} & lower class & 18.5 & 9.7 & 71.8 & 3,913 (37.6) & 10.7 & 89.3 & $6,503(36.8)$ \\
\hline & middle class & 16.1 & 8.9 & 74.9 & $2,103(20.2)$ & 9.6 & 90.4 & $3,582(20.3)$ \\
\hline & upper class & 12.7 & 8.8 & 78.4 & $4,396(42.2)$ & 6.6 & 93.4 & $7,577(42.9)$ \\
\hline \multirow[t]{2}{*}{ Belong to $\mathrm{IGO}^{\mathrm{b}^{* * *}}$} & not affiliated & 14.6 & 9.7 & 75.7 & $7,464(71.5)$ & 8.7 & 91.3 & $13,347(75.4)$ \\
\hline & affiliated & 18.1 & 7.8 & 74.1 & $2,970(28.5)$ & 8.8 & 91.2 & $4,350(24.6)$ \\
\hline \multirow{2}{*}{$\begin{array}{l}\text { Knowledge and } \\
\text { awareness }{ }^{\mathrm{ab}^{* * *}}\end{array}$} & none & 19.5 & 10.8 & 69.7 & $2,728(26.2)$ & 11.7 & 88.3 & 4,987 (28.2) \\
\hline & $\begin{array}{l}\text { knowledgeable } \\
\text { and aware }\end{array}$ & 14.2 & 8.6 & 77.2 & $7,684(73.8)$ & 7.6 & 92.4 & $12,675(71.8)$ \\
\hline \multicolumn{2}{|l|}{ Overall } & 15.6 & 9.2 & 75.2 & $10,434(100.0)$ & 8.7 & 91.3 & $\begin{array}{l}17,697 \\
(100.0)\end{array}$ \\
\hline
\end{tabular}

The results displayed in Table 4 show that marital status of women, exposure to mass media, religion, use of contraception and working status had no significance regarding the respondent's decision to use contraception as a method to prevent STDs and HIV/AIDS. Younger women aged $15-29$ years $(\mathrm{OR}=0.768,95 \% \mathrm{Cl}=0.662-0.891)$ made the decision to use contraception less often than their older counterparts. Hence, their husbands $(\mathrm{OR}=1.186,95 \% \mathrm{Cl}=$
0.996-1.413) were $18.6 \%$ more likely to make the decision of using contraception than women aged $40-49$ years.

The odds ratios of women living in Barisal, Chittagong and Khulna were 1.457, 0.771 and 1.316 , respectively. The results indicate that women residing in Barisal and Khulna were more likely to make the decision of using contraception than women living in the Sylhet division. Similarly, women living in urban areas made the decision regarding 
the use of contraception 1.141 times more frequently than women residing in the countryside, which is due to a large gap between rural and urban lifestyles.

The coefficient of education suggests that women who belong to below secondary educational category were $44.5 \%(\mathrm{OR}=1.445,95 \% \mathrm{Cl}=1.263-1.654)$ more likely to make the decision of using contraception than women with secondary + education. The results indicate that the level of education below secondary among both the respondents and their husbands/partners had a significant impact on the decision regarding the use contraception. Women from lower-class families committed to using contraception 1.159 times more often than women from upper-class households. Regardless of these factors, women who did not have any knowledge or awareness of STDs and HIV/AIDS made the decision of using contraception during sex 1.521 times more frequently than women who had some knowledge and awareness of STDs and HIV/AIDS.

Table 4 also reveals that the age of respondents, their marital status, religion and education of husband/partner had no significant impact on the right of women to refuse sex with an STD-infected husband. Women living in Barisal, Chittagong, Dhaka, Khulna and Rajshahi had 1.190, 1.457, 1.712, 1.980 and 3.258 times more capacity to refuse sex with their STD-infected husband, respectively, than women living in Sylhet. Likewise, women living in urban areas had $54.6 \%$

Table 4. Multinomial logistic regression model predictions of Odds Ratios (ORs) and Confidence Intervals (CI) for association between social determinants and behavior regarding STDs \& HIV

\begin{tabular}{|c|c|c|c|c|}
\hline \multirow[t]{2}{*}{ Social Determinants } & \multirow[t]{2}{*}{ Levels } & \multicolumn{2}{|c|}{ (\%) Decision for using contraception ${ }^{a}$} & \multirow{2}{*}{$\begin{array}{l}\text { (\%) Right to refuse sex: } \\
\text { husband has STD } \\
\text { Yes }\end{array}$} \\
\hline & & Respondent & Husband/Partner & \\
\hline \multirow[t]{3}{*}{ Age } & 15-29 years & $0.768(0.662-0.891)^{* * *}$ & $1.186(0.996-1.413)^{* *}$ & $0.975(0.843-1.127)$ \\
\hline & 30-39 years & $0.958(0.847-1.083)$ & $1.048(0.891-1.233)$ & $0.972(0.854-1.106)$ \\
\hline & 40-49 years (RC) & 1.000 & 1.000 & 1.000 \\
\hline \multirow[t]{2}{*}{ Marital status } & married (RC) & 1.000 & 1.000 & $0.964(0.759-1.224)$ \\
\hline & $\begin{array}{l}\text { separated/divorced } \\
\text { (RC) }\end{array}$ & - & - & 1.000 \\
\hline \multirow[t]{7}{*}{ Division } & Barisal & $1.457(1.156-1.836)^{* * *}$ & $0.860(0.662-1.116)$ & $1.190(0.983-1.441)^{*}$ \\
\hline & Chittagong & $0.771(0.605-0.983)^{* *}$ & $0.592(0.454-0.772)^{* * *}$ & $1.457(1.208-1.757)^{* * *}$ \\
\hline & Dhaka & $0.916(0.730-1.151)$ & $0.659(0.512-0.847)^{* * *}$ & $1.712(1.408-2.081)^{* * *}$ \\
\hline & Khulna & $1.316(1.054-1.644)^{* *}$ & $0.648(0.500-0.841)^{* * *}$ & $1.980(1.610-2.434)^{* * *}$ \\
\hline & Rajshahi & $0.897(0.712-1.129)$ & $0.542(0.415-0.708)^{* * *}$ & $3.258(2.556-4.153)^{* * *}$ \\
\hline & Rangpur & $1.021(0.814-1.281)$ & $0.527(0.403-0.688)^{* * *}$ & $0.935(0.783-1.117)$ \\
\hline & Sylhet (RC) & 1.000 & 1.000 & 1.000 \\
\hline \multirow[t]{2}{*}{ Place of residence } & urban & $1.141(1.003-1.297)^{* *}$ & $1.083(0.923-1.271)$ & $1.546(1.351-1.770)^{* * *}$ \\
\hline & rural (RC) & 1.000 & 1.000 & 1.000 \\
\hline \multirow[t]{2}{*}{ Education of women } & below secondary & $1.445(1.263-1.654)^{* * *}$ & $1.146(0.970-1.353)^{*}$ & $0.881(0.772-1.005)^{*}$ \\
\hline & secondary $^{+}(\mathrm{RC})$ & 1.000 & 1.000 & 1.000 \\
\hline \multirow{2}{*}{$\begin{array}{l}\text { Education of hus- } \\
\text { band/partner }\end{array}$} & below secondary & $1.411(1.229-1.621)^{* * *}$ & $0.952(0.805-1.126)$ & $1.076(0.944-1.226)$ \\
\hline & secondary $^{+}(\mathrm{RC})$ & 1.000 & 1.000 & 1.000 \\
\hline \multirow{2}{*}{$\begin{array}{l}\text { Exposure to mass } \\
\text { media }\end{array}$} & no access & $1.019(0.889-1.167)$ & $1.400(1.176-1.667)^{* * *}$ & $0.781(0.685-0.891)^{* * *}$ \\
\hline & access (RC) & 1.000 & 1.000 & 1.000 \\
\hline \multirow[t]{2}{*}{ Religion } & muslim & $1.067(0.892-1.277)$ & $1.095(0.870-1.378)$ & $1.039(0.872-1.238)$ \\
\hline & others (RC) & 1.000 & 1.000 & 1.000 \\
\hline \multirow[t]{2}{*}{ Use of contraceptive } & not using & 1.000 & 1.000 & $898(0.802-1.007)^{*}$ \\
\hline & $\begin{array}{l}\text { using any method } \\
\text { (RC) }\end{array}$ & - & - & 1.000 \\
\hline \multirow[t]{2}{*}{ Working status } & not working & $0.944(0.842-1.060)$ & $1.377(1.177-1.610)^{* * *}$ & $0.894(0.793-1.010)^{*}$ \\
\hline & working (RC) & 1.000 & 1.000 & 1.000 \\
\hline \multirow{3}{*}{ Economic status } & lower class & $1.159(0.980-1.372)^{*}$ & $1.000(0.808-1.238)$ & $0.907(0.770-1.068)$ \\
\hline & middle class & $1.121(0.951-1.321)$ & $1.016(0.827-1.247)$ & $0.844(0.721-0.989)^{* *}$ \\
\hline & upper class (RC) & 1.000 & 1.000 & 1.000 \\
\hline \multirow[t]{2}{*}{ Belong to IGO } & not affiliated & $0.789(0.704-0.885)^{* * *}$ & $1.223(1.046-1.429)^{* *}$ & $0.829(1.013-0.898)^{* *}$ \\
\hline & affiliated (RC) & 1.000 & 1.000 & 1.000 \\
\hline \multirow{2}{*}{$\begin{array}{l}\text { Knowledge and } \\
\text { awareness }\end{array}$} & none & $1.521(1.355-1.708)^{* * *}$ & $1.390(1.200-1.609)^{* * *}$ & $0.616(0.553-0.687)^{* * *}$ \\
\hline & $\begin{array}{l}\text { knowledgeable and } \\
\text { aware (RC) }\end{array}$ & 1.000 & 1.000 & 1.000 \\
\hline
\end{tabular}


$(\mathrm{OR}=1.546,95 \% \mathrm{Cl}=1.351-1.770)$ more capacity to refuse sex with an STD-infected husband than women residing in the countryside. Urban women thus possessed greater decision making capacity to copulate with an STD-infected husband/partner.

Women who belong to the educational category below secondary had 0.881 times less capacity to refuse sex with their STD-infected husband than women whose level of education was secondary+. Similarly, women who were not working had 0.894 times less capacity to refuse sex with their STD-infected husband than women who were employed. Middle-class women $(\mathrm{OR}=0.844,95 \% \mathrm{Cl}=0.721-0.989)$ were less likely to refuse sex with their STD-infected husband than women from upper-class families. Therefore, education, working and economic status of women determine their capacity to refuse sex with their STD-infected husband, which is crucial to avoid the transmission of STDs and HIV. Women who were not knowledgeable and aware of STDs and HIV/AIDS had less capability to refuse sex with their STD-infected husband than educated and informed women.

\section{Discussion}

The purpose of the study was to evaluate the influence of social factors on the level of knowledge and awareness about STDs and HIV/AIDS among women and their sexual behaviors. Working on the assumption that social factors do affect women's knowledge and awareness of STDs and HIV/AIDS, especially among women aged 15-29 years, compared to their older counterparts, is consistent with results of other prior studies conducted in Bangladesh [16]. Young women were less likely to make the decision of using contraception than their older counterparts. They also had lower capacity to refuse sex with their STD-infected husband/partner, although the difference was not statistically significant. The traditional social system, lack of women's empowerment, as well as a low level of knowledge of sexual and reproductive health are responsible for this situation; this is primarily because younger women $(<29$ years) are generally perceived as being neither children nor adults. This situation can be improved by changing the traditional social system [16, 17], along with the introduction of adult health and sexual education programs at the population level.

Geographical location was an important social determinant influencing the level of knowledge and awareness of STDs and HIV/AIDS among women. Women living in Dhaka, Khulna, Barisal and Chittagong had more comprehensive knowledge and awareness regarding STDs and HIV/ /AIDS and adopted safer sexual practices, which was due to their level of higher education and exposure to multiple sources of information on sexual and reproductive health issues. In contrast, women living in Sylhet, Rangpur and Rajshahi had poor knowledge and awareness and engaged in riskier behavior due to lack of education and information, religiosity, conservative attitude and traditional mindset. Hence, there is a wide gap between women living in rural and urban areas regarding the use of contraception and the capacity to refuse sex with an STD-infected husband/partner. Rural women are less educated, have a lower degree of sexual autonomy and limited access to mass media along with information on STDs and HIV/AIDS than women living in urban areas, which is close to the results of other studies $[9,16,17]$. In this regard, the level of knowledge and awareness of STDs and HIV/AIDS (and their risk-free outlook) could be raised by reducing geographical disparities.

Education of women and their partners/husbands had a profound impact on the level of knowledge and awareness about STDs and HIV/AIDS among women. The differences in the level of knowledge and awareness were statistically significant; this result was in agreement with prior research in Bangladesh and other countries [9, 15-18]. Education broadens our horizons and makes it easier for us to access information on sexual and reproductive health from various communication channels, including the Internet, television, radio, print media, social media and opinion leaders. It is difficult for uneducated people to comprehensively decode information on STDs and HIV/AIDS; thus, the level of education is a significant determinant of knowledge, awareness and behavior regarding STDs and HIV/AIDS. Our findings correspond to the results of other studies in this respect $[9,16]$. A woman's sexual autonomy to make decisions regarding the use of contraception and their capacity to refuse sex with an STD-infected husband/ /partner are also heavily dependent on the level of education of both women and their partners/husbands. Therefore, a higher level of education of women and their partners has a positive influence on women's sexual practices.

Exposure to mass media, religious background and the use of contraception have a substantial impact on the level of knowledge and awareness of STDs and HIV/AIDS, as well as safe sexual practices. The study revealed that exposure to mass media significantly increased the level of comprehensive knowledge and awareness of STDs and HIV/ /AIDS. It strongly affected a woman's capacity to refuse sex with an STD-infected husband/partner, as radio, television and newspapers are the main sources of information about STDs and HIV/AIDS. If women were not exposed to mass media, it was generally their husband's/partner's decision as to whether or not contraception was used. Apart from traditional mass media, new media, as well as social media platforms like Facebook, Twitter, etc., can be a great source of information about STDs and HIV/AIDS and may shape a woman's positive behavior, due to their current popularity and broad reach. Our study indicated that Muslim women had comprehensive knowledge about STDs and HIV/AIDS, but this has an insignificant effect on their decision to use contraception or to refuse sex with an STD-infected partner/ /husband. Women who did not use contraception did not have enough sexual autonomy to refuse sex with an STDinfected husband/partner, as they may not be empowered to make this decision. These findings bear resemblance to the results of other studies [9, 15-19].

The employment and economic status of women were crucial social determinants of the level of knowledge and awareness of STDs and HIV/AIDS and safe sexual practices. Unemployed and lower-class women had poor knowledge and awareness of STDs and HIV/AIDS, while better-educated women, as well as women from the middle or upper class, demonstrated greater degree of knowledge regarding STDs. Women's employment and economic strength ensure their sexual autonomy to make the decision of using contraception and the capacity to refuse sex with an STD-infected husband/ /partner. Equal opportunities for education, employment and financial solvency ensure comprehensive knowledge and awareness of STDs and HIV/AIDS and promote risk-free sexual behavior regarding STDs and HIV/AIDS [22-35]. Being affiliated with an IGO had no impact on the level of knowledge and awareness, due to their excessive focus on microcredit lending rather than providing basic education, healthcare and social awareness, which should be part of their services [39]. In contrast, affiliation to an IGO empowered women to make the decision of using contraception and refusing sex with an STD-infected husband/partner. IGOs lend money only to women, who subsequently motivate men to consider the woman's opinion during sexual intercourse.

A higher level of knowledge and awareness of STDs and HIV/AIDS encourages safe sexual practices and promotes risk-free behaviors. Women who had no knowledge and awareness about STDs and HIV/AIDS engaged in unsafe sex and risky sexual behaviors. Women did not refuse to have 
sex with their STD-infected husband/partner if they did not have knowledge and awareness about STDs and HIV/AIDS. Several similar studies also indicated that knowledge and awareness of STDs and HIV/AIDS reduce the risk of sexually transmitted infections [9-13, 15-21].

The study showed that social determinants such as age, geographical location, women's and their husbands'/partners' level of education, exposure to mass media, religion, employment and economic status had a substantial impact on the level of knowledge and awareness, as well as sexual behaviors, regarding STDs and HIV/AIDS. These are responsible for the development and the re-emergence of STDs and HIV/AIDS and are also highly associated with the social epidemiology and social determinants health approach [23-32]. Evidence shows that the level of knowledge and awareness about STDs and HIV/AIDS varies significantly depending on social determinants. For instance, women from lower-class families, less educated, unemployed and living in rural areas demonstrated poor knowledge about STDs and HIV/AIDS, had less autonomy in their decision for the use of contraception and had less capacity to refuse sex with an STD-infected husband/partner. Results of the study indicate that rural-urban disparities, geographical location, unequal economic and unemployment status, poor education and lack of knowledge proliferate the risk of the HIV/AIDS epidemic. Previous studies show similar results [30, 40]. A fully effective cure for HIV/AIDS is unavailable, and there is a significant influence of social determinants on the prevalence of this infectious disease. Therefore, the results suggest that more attention must be focused on the underlying social determinants of the disease in order to prevent the spread of HIV/AIDS [30, 34-35, 40, 41]. Consequently, removing social inequalities, fighting social exclusion, as well as promoting social and economic development, is a precondition for ending the AIDS epidemic by 2030 as part of SDGs [34, $35,41]$. A more comprehensive social policy to fight social inequalities will be crucial in combating the spread of STDs and HIV/AIDS worldwide, as well as in Bangladesh [41].

\section{Conclusions}

The research showed that the level of knowledge and awareness about STDs and HIV/AIDS among women remained unchanged throughout the period from 2011 to 2014, due to social disparities among women. The study disclosed that social determinants have a considerable impact on the level of knowledge and awareness about STDs and HIV/AIDS, as well as promote risk-free sexual behavior. The study has addressed the social epidemiological model and the WHO social determinants model of health. The knowledge, awareness and behavior associated with STDs and HIV/AIDS were self-reported responses of women and cross-sectional in nature. In this regard, an in-depth study could be a possible method of comprehensive interpretation. Furthermore, the study did not include single women, though this could be a valuable addition. Despite a few limitations, the research has provided a consistent argument that social determinants influence the knowledge, awareness and behavior of women regarding STDs and HIV/AIDS. This study advocates initiating more programs to eliminate social disparities among women by incorporating formal and informal health promotion into the educational program and launching mass media health campaigns targeting women. The Ministry of Health can support IGOs and urge them to initiate STD and HIV/AIDS-oriented programs at the local level. Family doctors can educate their patients regarding STDs and HIV/ /AIDS, as well as promote risk-free behaviors to eliminate sexually transmitted infections. Public health policy makers and planners must consider the social determinants of health and integrate it into both local and global HIV/ AIDS reduction strategy in order to come out on top of this pernicious epidemic.

Acknowledgments. The author recognizes the BDHS, NIPORT and ICF for their approval to use this data set. The author acknowledges Dr. Hasan Mahmud for his scholarly comments. In addition, the author is grateful to the reviewers for their comments and suggestions on the further improvement of this manuscript. Author also thanks to Shahriar Khandaker and Toufica Sultana for their assistance.

Source of funding: This work was funded by the author's resources. Conflict of interest: The author declares no conflict of interests.

\section{References}

1. Lazarus JV, Sihvonen-Riemenschneider H, Laukamm-Josten U, et al. Systematic review of interventions to prevent the spread of sexually transmitted infections, including HIV, among young people in Europe. Croat Med J 2010; 51(1): 74-84, doi: 10.3325/ cmj. 2010.51.74.

2. Joint United Nations Programme on HIV/AIDS. AIDS by the numbers, 2015 [cited 30.03.2016]. Available from URL: http:// search.unaids.org, doi: JC2571/1/E.

3. United Nation Population Division. World Population Prospects The 2012 Revision: Highlights and Advance Tables, 2013 [cited 30.03.2016]. Available from URL: http://esa.un.org/unpd/wpp/.

4. National Institute of Population Research and Training, Mitra and Associates, and ICF International. Bangladesh Demographic and Health Survey [cited 30.03.2016]. Available from URL: http://www.niport.gov.bd/wp-content/uploads/publication/1432536472BDHS\%202014\%20KIR.pdf.

5. United Nations Population Fund. United Nations Family Planning Assistance: Making one billion counts [cited 30.03.2016]. Available from URL: http://www. advocatesforyouth.org/component/content/article/1648-one-billion-dollars-for-us-international-family-planning-assistance.

6. Svensson L. Knowledge of and attitudes to sexually transmitted diseases among Thai university students, Uppsala University, Sweden, 2013 [cited 30.03.2016]. Available from URL: http://www.diva-portal.org/smash/get/diva2:632901/FULLTEXT01.pdf.

7. World Health Organization, Health for the World's Adolescents A second chance in the second decade, Geneva, 2014 [cited 30.03.2016]. Available from URL: http://apps.who.int/adolescent/second-decade/files/1612_MNCAH_HWA_Executive_Summary.pdf.

8. Mathers CD, Loncar D. Updated projections of global mortality and burden of disease, 2002-2030: data sources, methods and results, Geneva, 2005 [cited 30.03.2016]. Available from URL: http://www.who.int/healthinfo/statistics/bod_projections2030_ paper.pdf.

9. Khan MA. Knowledge on AIDS among female adolescents in Bangladesh: evidence from the Bangladesh demographic and health survey data. J Health Popul Nutr 2002; 20(2): 130-137 [cited 30.03.2016]. Available from URL: http://www.ncbi.nlm. nih.gov/pubmed/12186193.

10. Babul P. Bangladesh is at high risk of HIV/AIDS epidemic. Star Mag 2010; 47: 22-23.

11. Azim T, Islam MN, Bogaerts J, et al. Prevalence of HIV and syphilis among high-risk groups in Bangladesh. AIDS 2007; 14(2): $10-11$. 
12. Shah SA, Kristensen S, Khan OA. Behavioral and biomedical risk factors for the transmission of HIV/AIDS in Bangladesh. Int J STD AIDS 2000; 11(2): 133-134.

13. Gibney L, Choudhury P, Khawaja Z, et al. Behavioral risk factors for HIV/AIDS in a low-HIV prevalence Muslim Nation: Bangladesh. Int J STD AIDS 1999; 10(3): 186-194.

14. World Bank. Bangladesh HIV/AIDS Update: Regional Update, South Asia Region (SAR)-Bangladesh 2010, [cited 30.03.2016]. Available from URL: http://www.worldbank.org/en/news/feature/2012/07/10/hiv-aids-bangladesh.

15. Khan MA, Rahman M, Khanam PA, et al. Awareness of sexually transmitted diseases among women and service providers in rural Bangladesh. Int J STD AIDS 2008; 8(11): 688-696.

16. Hossain M, Mani KK, Sidik SM, et al. Knowledge and awareness about STDs among women in Bangladesh. BMC Public Health 2014; 14: 775, doi: 10.1186/1471-2458-14-775.

17. Hasan ATM H, Hassan R, Khan ZR, et al. Influence of socio-demographic factors on awareness of HIV/AIDS among Bangladeshi garment workers. Springer Plus 2013; 2: 174 [cited 30.03.2016]. Available from URL: http://www.springerplus.com/ content/2/1/174 doi: 10.1186/2193-1801-2-174.

18. Kaptanoğlu AF, Süer K, Diktaş H, et al. Knowledge, attitudes and behaviour towards sexually transmitted diseases in Turkish Cypriot adolescents. Cent Eur J Public Health 2013; 21(1): 54-58.

19. Jesmin SS, Chaudhuri S, Abdullah S. Educating women for HIV prevention: does exposure to mass media make them more knowledgeable? Health Care Women Int 2013; 34(3-4): 303-331, doi: 10.1080/07399332.2012.736571.

20. Gibney L, Saquib N, Metzger J. Behavioral risk factors for STD/HIV transmission in Bangladesh's trucking industry. Soc Sci Med 2003; 56(7): 1411-1424.

21. Islam M, Conigrave KM. Review HIV and sexual risk behaviors among recognized high-risk groups in Bangladesh: need for a comprehensive prevention program. Int J Infect Dis 2008; 12(4): 363-370, doi: 10.1016/j.ijid.2007.12.002.

22. Putnam S, Galea S. Epidemiology and the macrosocial determinants of health. J Public Health Policy 2008; 29(3): 275-289, doi: 10.1057/jphp.2008.15.

23. Berkman LF, Kawachi I. A historical framework for social epidemiology. In: Berkman LF, Kawachi I, eds. Social epidemiology. New York: Oxford University Press; 2000.

24. Cassel J. The contribution of the social environment to host resistance: the fourth Wade Hampton Frost lecture 1976. Am J Epidemiol 1995; 104(2): 107-123.

25. Galdston I. Social medicine: its derivations and objectives. New York: Commonwealth Fund; 1949.

26. Krieger N. Theories for social epidemiology in the 21 st century: an ecosocial perspective. Int J Epidemiol 2001; 30(6): 68-77.

27. Marmot MG. Stress, social and cultural variations in heart disease. J Psychosom Res 1983; 27(5): 377-384.

28. Marmot M. Socioeconomic determinants of CVD mortality. Int J Epidemiol 1989; 18(3), Suppl 1: S196-S2O2.

29. Syme SL. Historical perspective: the social determinants of disease-some roots of the movement. Epidemiol Perspect Innov 2005; 2(2), doi: 10.1186/1742-5573-2-2.

30. Farmer P. Social inequalities and emerging infectious diseases. Emerg Infect Dis 1996; 2: 259-69.

31. World Health Organization, Commission on Social Determinants of Health. A Conceptual Framework for Action on the Social Determinants of Health (Draft). 2007. Avaible from URL: http://www.who.int/social_determinants/resources/csdh_framework_action_05_07.pdf.

32. Marmot M. Public Health Social determinants of health inequalities. Lancet 2005; 365(9464): 1099-1104, doi: 10.1016/S01406736(05)71146-6.

33. Miller C, Waning B, Beard J, et al. Social epidemiology. In: Rickles NM, Wertheimer AI, Smith MC, eds. Social and behavioral aspects of pharmaceutical care. Burlington: Jones \& Bartlett Publishers; 2009.

34. Bates I, Fenton C, Gruber J, et al. Vulnerability to malaria, tuberculosis, and HIV/AIDS infection and disease. Part II: Determinants operating at environmental and institutional level. Lancet Infect Dis 2004; 4(6): 368-375.

35. Ali SH. A socio-ecological autopsy of the E. coli O157:H7 outbreak in Walkerton, Ontario, Canada. Soc Sci Med 2004; 58(12): 2601-2612.

36. The United Nations Children's Fund. Bangladesh statistics 2009 [cited 30.03.2016]. Available from URL: http://www.unicef. org/infobycountry/bangladesh_bangladesh_statistics.html.

37. Joint United Nations Programme on HIV/AIDS (UNAIDS), Fast-Track: Ending AIDS epidemic by 2030, Geneva 2014 [cited 30.03.2016]. Available from URL: http://www.unaids.org/sites/default/files/media_asset/JC2686_WAD2014report_en.pdf.

38. Lattin J, Carrol JD, Green PE. Analyzing multivariate data. Pacific Grove, CA: Thomson; 2003: 474-495.

39. Hadi A. Integrating prevention of acute respiratory infections with micro-credit programme: experience of BRAC, Bangladesh. Public Health 2002; 116(4): 238-244.

40. Malarcher S, eds. Social determinants of sexual and reproductive health: informing future research and programme implementation. Geneva: WHO Press; 2010.

41. Johnston HB. Relationships of exclusion and cohesion with health: the case of Bangladesh. J Health Popul Nutr 2009; 27(4): 426-440, doi:10.3329/jhpn. v27i4.3387.

Tables: 4

Figures: 1

References: 41

Received: 23.10 .2016

Revised: 22.11 .2016

Accepted: 22.11.2016

Address for correspondence:

Juwel Rana

Executive Director, South Asian Youth Research Institute for Development, Bangladesh

House-23/24, Block-C

Tajmahal Road, Mohammadpur, Dhaka

Bangladesh

Tel.: +8801737220627

E-mail: juwelrana@sayrid.org

Personal Web-page: www.juwelrana.com 